Published in final edited form as:

Nat Biomed Eng. 2017 ; 1: . doi:10.1038/s41551-017-0083.

\title{
3D-printed vascular networks direct therapeutic angiogenesis in ischaemia
}

\author{
T. Mirabella ${ }^{1}$, J.W. MacArthur ${ }^{2}$, D. Cheng ${ }^{1}$, C.K. Ozaki ${ }^{3}$, Y.J. Woo ${ }^{4}$, M. Yang ${ }^{5}$, and C.S. \\ Chen ${ }^{1, *}$ \\ ${ }^{1}$ Department of Bioengineering and the Biological Design Center, Boston University; The Wyss \\ Institute for Biologically Inspired Engineering, Harvard University, Boston, MA \\ ${ }^{2}$ Department of Surgery, University of Pennsylvania, Philadelphia, PA \\ ${ }^{3}$ Department of Surgery, Brigham and Women's Hospital and Harvard Medical School, Boston, \\ MA \\ ${ }^{4}$ Department of Cardiothoracic Surgery, Stanford University, Palo Alto, CA \\ ${ }^{5}$ Innolign Biomedical, Boston, MA
}

\begin{abstract}
Arterial bypass grafts remain the gold standard for the treatment of end-stage ischaemic disease. Yet patients unable to tolerate the cardiovascular stress of arterial surgery or those with unreconstructable disease would benefit from grafts that are able to induce therapeutic angiogenesis. Here, we introduce an approach whereby implantation of 3D-printed grafts containing endothelial-cell-lined lumens induces spontaneous, geometrically guided generation of collateral circulation in ischaemic settings. In rodent models of hind-limb ischaemia and myocardial infarction, we demonstrate that the vascular patches rescue perfusion of distal tissues, preventing capillary loss, muscle atrophy and loss of function. Inhibiting anastomoses between the construct and the host's local capillary beds, or implanting constructs with unpatterned endothelial cells, abrogates reperfusion. Our 3D-printed grafts constitute an efficient and scalable approach to engineer vascular patches able to guide rapid therapeutic angiogenesis and perfusion for the treatment of ischaemic diseases.
\end{abstract}

Ischaemic cardiovascular disease remains the leading cause of morbidity and mortality in the United States and continues to grow at an alarming rate worldwide $(1,2)$. Impaired blood circulation to different organs and tissues underlies debilitating conditions such as limb

\footnotetext{
Users may view, print, copy, and download text and data-mine the content in such documents, for the purposes of academic research, subject always to the full Conditions of use: http://www.nature.com/authors/editorial_policies/license.html\#terms

*Correspondence should be directed to chencs@bu.edu.

Author contributions: T.M., J.W.M., D.C., K.C.O, J.W.Y, M.Y. and C.S.C. conceived, developed and mentored the project. T.M., J.W.M., D.C. and M.Y. performed experiments. T.M. and J.W.M, analyzed the data. T.M. and C.S.C. wrote the manuscript.

Competing interests: C.S.C. is a cofounder of, and owns equity in, Innolign Biomedical, a company that is developing tissue engineered products.
}

Data Availability

The authors declare that all data supporting the findings of this study are available within the paper and its supplementary information. 
ischaemia, heart failure and stroke (3-5). While percutaneous revascularization and artery bypass surgery continue to remain mainstay treatments for alleviating ischaemia in large vessel occlusive artery disease, the generation of collateral circulation via the recruitment of small vessels, or therapeutic angiogenesis, could offer an additional (even adjunctive) approach that would address an unmet need. Traditional open surgical bypass is not well tolerated in many ischaemic patients due to co-morbidities that drive peri-procedural complications (6-11), and anatomic and durability barriers continue to limit more widespread application of endovascular therapies. Furthermore, in many instances disease is not limited to large arteries and additional approaches are needed to enhance perfusion at the level of the microvasculature (12-14).

Initial efforts to develop pro-angiogenic therapies focused on the delivery of angiogenic growth factors (e.g. VEGF, FGF) to an ischaemic site (15-17). However, it was quickly realized that such soluble factors are rapidly extracted and eliminated from a site. Moreover, the disappointing results of pro-angiogenic VEGF therapy have been linked to a significant increase in edema (18). Subsequent attempts used controlled-release biomaterials or expression vectors delivered to either local cells or injected engineered cells $(15,19-22)$. Cytokine and cell based micro-revascularization showed remarkable initial experimental promise, but then clinically insignificant benefits in prospective randomized clinical trials (23). Recent studies in rodents have shown that injection of vascular endothelial cells and stromal cells attract host vessels to invade into a site to form new vessels. However, the invasion process takes weeks and the resultant vasculature is randomly organized unlike native capillary networks $(24,25)$. Thus, no current therapies to restore microcirculation have reached mainstream clinical practice.

Within the tissue engineering field, recent studies have demonstrated that pre-organizing endothelial cells into defined network architectures within small tissue engineered constructs can significantly enhance the speed and extent of vascularization of the construct upon implantation (26-28). However, the extent to which such an approach could be scaled and adapted to treat ischaemia of endogenous mammalian tissues remains unclear.

Here, we present an approach using 3D printing to pre-organize endothelial cells into patterned channels within a fibrin patch, which upon implantation, facilitated rapid invasion and integration of small collaterals from the host to generate perfused, functional blood vessels that mirrored the architecture of the implanted grafts. By using constructs containing long, centimetre-scale channels oriented in parallel as means to direct revascularization from well-perfused sites into surrounding ischaemic tissues, we demonstrate the potential utility of the approach in two relevant models of ischaemic disease.

\section{Results}

\section{D printed vascular patches functionally engraft in vivo and rescue perfusion and function of ischaemic tissues}

In this study, we set out to test whether organizing endothelial cells into patterned channels within an implantable, fully degradable fibrin patch would enhance perfusion in rodent models of ischaemia. To engineer endothelial networks, we used a recently described 
method to 3D print a network of sugar filaments into a network, encapsulate the network in a fibrin gel, dissolve the sugar to leave behind channels, and then inject endothelial cells to fill the channels (Fig. 1a) (29); To assess in vivo the efficacy and translational value of these patterned constructs, we implanted them in a mouse model of hind limb ischaemia and tracked perfusion of distal limbs from 5 to 21 days. In this model, occlusion of the left femoral artery distal to the inferior epigastric and profunda femoris arteries (injury site) caused a 50\% post-operative decrease in perfusion of the distal ischaemic limb as measured by laser Doppler imaging (Fig. 1b). In mice implanted at the injury site with vascular patches (VP), perfusion measured distally at the feet progressively increased over time, to reach the value of the contralateral, non-ischaemic limb by 5 days post operatively (Fig. 1b). In the sham group, perfusion did not substantially improve from the post-operative levels.

Ischaemia experienced in the limb distal to the site of artery occlusion induced, as expected, an angiogenic response in the gastrocnemius (GC) muscle of the lower limb, as indicated by the number of vessels normalized to the number of muscle fibres (Fig. 1c). The number of capillaries normalized to the number of muscle fibres, was abnormally high in the sham group compared to the VP group (Fig. 1d). Upon closer examination of histologic sections, we also observed a substantial increase in apoptotic ECs within capillaries of the sham group but not in the VP group. In VP implanted mice, muscle fibres appeared healthy with a characteristic distribution of diameters (Fig. 1d). While in the sham group, there was observed degeneration of muscle tissue. Fibres were smaller $\left(\mathrm{area}<1800 \mathrm{um}^{2}\right)$ and showed a diffuse hypercellularity indicative of early fibrosis (Fig. 1e). Thus the implanted vascular patches appeared to restore perfusion to distal tissues and rescue ischaemic muscle damage.

\section{Direct anastomoses of vascular patches with host vasculature drives functional perfusion}

To better understand how the vascular patches restored distal perfusion, we examined the vasculature at the site of implantation. Laser Doppler imaging revealed progressively improved perfusion both up- and downstream of the VP by day 3 , suggestive of the establishment of collateral circulation through the construct (Suppl. Fig. 1). Upon retrieval at 5 days, approximately two thirds of the endothelialized channels originally implanted were visibly present in constructs, with the attrition likely due to partial resorption of the fibrin patch. Histology of the explanted construct confirmed the presence of host capillary vessels directly connecting to the pre-patterned endothelial channels, with new smooth muscle actinpositive mural cells lining the patterned vessels and blood perfused within them (Fig. 2a). To further confirm perfusion of channels via direct anastomosis to the systemic circulation, dyelabeled dextran was perfused systemically by tail vein injection minutes before tissue explantation. The presence of dye within the channels confirmed perfusion by the host vasculature (Fig. 2a). About $90 \%$ of the retrieved channels were functional (dextran perfused). Labeling with monoclonal anti-mouse CD31 revealed that these capillaries were of host origin at the interface between the implant and the surrounding tissue (Fig. 2b). Long and branched capillaries transited across the implant and reached the patterned construct, lining the edges of the channels. The vascular walls of the patterned vasculature were found to be chimerically occupied by both implanted endothelial cells and host endothelial cells, and coupled to host capillaries. EphrinB2 and EphB4 staining further confirmed that most endothelial cells within the microchannels expressed the vein marker EphB4, and only a 
small subset of endothelial cells expressed ephrinB2 consistent with the nature of the implanted HUVECs and host capillary endothelial cell invasion (Suppl Fig. 2).

While connectivity between host and implant vasculature could be critical to the therapeutic effect of the constructs, another possibility is that the implanted endothelial cells simply provide paracrine support for host vasculature. To test this, we implanted constructs containing endothelial cells in which the expression of the sprout-inducing GTPase, CDC42, was silenced by siRNA. SiRNA treated cells (siCDC42) were confirmed to lack expression of $\mathrm{CDC} 42$, reduced invasive sprouting in vitro, but retain normal viability (Suppl Fig. 3). In vivo, siCDC42 versus control VP revealed substantially reduced integration between host capillaries and patterned engineered vasculature (Fig. 2c), with less vessels arising from the implanted VP (parent vessels) and lower anastomotic connections between parent and host vessels. Channels where CDC42 was silenced still recruited host mural cells, though with decreased coverage (Fig. 2d). Anastomosis between channels and host capillaries were fewer in number, with systemically injected lectin still found within the channel walls (Fig. 2d). However, at a functional level, LDI showed a significant decrease of distal perfusion to ischaemic limbs in mice implanted with siCDC42 VP, compared to scrambled control VP (Fig. 2e). Thus, bio-integration of vascular graft and functional recovery result from local connection between host and implant vasculature to give rise to a functionally significant collateral circulation.

\section{Patterning of vascular architecture is critical to therapeutic benefit}

To clarify whether the therapeutic effect of the implant was merely due to the presence of the exogenous endothelial cells secreting angiocrine cues, or rather ECs patterned in a certain architecture, we compared constructs containing endothelialized channels in vascular patches (VP, as before) with constructs carrying the same number of endothelial cells randomly embedded into fibrin not patterned with channels (endothelial patches, EP) and with fibrin patterned with channels but not seeded with cells (acellular patches, AP). Mice implanted with constructs lacking cells (AP) or organized features (EP) were not able to rescue perfusion of the ischaemic limb and were comparable to non-treated shams, even in 21 days long term studies (Fig. 3a, Suppl. Fig. 4). Moreover, the histology of EP confirmed a lack of substantial invasion or anastomosis of host vessels with implant vessels as compared to the patterned constructs (Suppl. Fig. 5). These results suggest that merely the presence of endothelial cells without concomitant organization into vessel-like structures fails to establish the organized collateral circulation needed to perfuse distal ischaemic sites.

We next examined whether changes in the geometry of the vascular channels, rather than their presence or absence, could impact their therapeutic function. Decreasing the diameter of the 3D printed channels from $400 \mu \mathrm{m}$ (VP) to $200 \mu \mathrm{m}$ (Sm_D VP), while keeping constant the amount of HUVECs seeded (e.g., by increasing the number of channels per patch), decrease the ability of the patch to rescue perfusion of distal limbs to the extent that the effect was no longer significantly different from negative control groups (EP and AP). We also generated constructs where microchannels were arranged in a grid shape (Grid VP) compared to our standard constructs where microchannels run parallel to each other (Par VP), keeping constant the number of channels present in each patch. Grid VP implants 
exhibited reduced efficacy in rescuing perfusion compared to Par VP to the extent that they were not significantly different from negative control groups. Together, these results suggest that the geometric design of the vascular channels within the patch impacts its ability to rescue perfusion, perhaps by affecting the efficiency of directing blood volume and flow from non-ischaemic to ischaemic regions, a parameter critical for recovery of distal perfusion and function.

\section{D printed vascular patches rescue cardiac function in a model of myocardial infarction}

Given the potential utility of VP to rescue perfusion in the ischaemic limb, we then sought to investigate whether the approach could improve outcomes in the more demanding setting of cardiac infarction, where perfusion deficits lead to irreversible damage. A well-defined myocardium infarction (MI) was generated by ligation of the left anterior descending coronary artery in athymic rats (30-34). Constructs patterned with aligned channels of endothelial cells (VP) were placed on the surface of the heart spanning over the border between non-ischaemic and ischaemic zones of the underlying heart (Fig. 4a). Healthy rats (no MI) and infarcted rats receiving saline injections following artery ligation (Sham) were used as treatment controls. At 4 weeks after the surgeries, both non-invasive functional measurements and terminal histology assessment were performed in the ischaemic region of the rat hearts. Gross analysis of the explanted rat hearts revealed increased vascularization throughout the implant and surrounding native tissue. The vasculature within the explanted patch was comprised of dense networks of host capillaries connecting with long segments of parent channels, which were functionally integrated as they were DiI perfused after systemic infusion (Fig. 4a). Immunohistochemical staining of vWF positive vessels in the ischaemic zone of cardiac tissue indicated loss of capillaries in untreated infarcted hearts compared to healthy controls, and a substantial increase in capillary densities following treatment with VP (Fig. 4b). Aortic flow probe and intraventricular pressure-volume conductance measurements were obtained to determine cardiac output and other functional parameters (Fig. 4c-e). Analysis of the measurements indicated that hearts from the sham group exhibited half the ejection fraction and cardiac output of no MI controls (Fig. 4d,e). In contrast, VP resulted in partial rescue of cardiac function, with an ejection fraction and a cardiac output close to that measured in healthy controls (Fig. 4d,e). Moreover, using transthoracic echocardiographic analysis, the left ventricular internal dimension could be non-invasively measured for hearts in diastole (LVIDd) and systole (LVIDs) (Fig. 4f). Untreated ischaemic hearts exhibited substantial increases in LVIDd and LVIDs compared to healthy controls, while rats treated with VP implants substantially did not develop significant ventricle dilation (Fig. 4f). Together, these results demonstrate substantial functional rescue of cardiac function following a major ischaemic injury.

\section{Discussion}

Previous work has demonstrated that one can induce robust elaboration of capillary beds simply by injecting a variety of pro-angiogenic growth factors, gene therapy vectors, cells, and/or biomaterials (15-22). Here, by implanting a patch containing pre-engineered vessels, we not only observed enhanced angiogenesis, but also the engineered vessels became integrated with the host vasculature and resulted in significant perfusion of distal ischaemic 
tissues. The demonstration that the therapeutic effect required anastomosis between host and engineered vasculature suggests that the larger calibre (150-400 $\mu \mathrm{m}$ diameter) engineered vessels may significantly enhance the volume of blood delivered distally. Indeed, to deliver blood across centimetre distances only via the highly branched, cascading structure of capillary beds is biomechanically highly inefficient $(35,36)$. It is thus not entirely surprising that we observed that constructs containing disorganized endothelial cells-previously shown to give rise to highly branched capillary beds (25) —-fail to rescue sufficient circulation to meet the demands of a major artery. The fact that capillaries may not be well suited to address ischaemia, except in cases where low capillary density is the primary limitation to tissue perfusion, could contribute to the lack of success of therapeutic angiogenesis in clinical ischaemia despite promise in small rodent studies. Nevertheless, further studies in larger animal models demanding rescue of perfusion across larger scale ischaemic territories will be required in order to evaluate whether the strategy of implanting oriented patterns of engineered vessels would ultimately have therapeutic merit at the clinical scale.

Additive manufacturing, or 3D printing, has rapidly advanced as an approach to fabricate numerous types of complex structures across research and industry sectors (37). 3D printing has been adopted to fabricate various types of engineered tissue structures, primarily through the use of low-pressure nozzles to print natural or synthetic hydrogels in the presence or absence of suspended cells (38). We and others have recently extended these efforts by using $3 \mathrm{D}$ printing to generate microvessel networks in vitro to allow one to model or grow thicker tissues that require perfusion $(29,39)$. Here, by using 3D printing to generate patterns of vasculature in vitro, we are able to provide a path for engineering host vasculature in vivo. Given that vascular architecture follows specific patterns conserved across many species $(35,36)$, but is also distinct in different tissue settings, it is not surprising that our studies demonstrate that geometry of the channels is a design parameter that impacts functionality of the implant, and we anticipate that the pattern of engineered vasculature used in constructs could dramatically impact its performance in promoting circulation in different contexts. Thus, this vascular guidance technology could provide a means not only to re-wire circulatory pathways for a variety of applications, but that each application may need to be customized with different architectural and functional outcomes depending on the host vascular context.

Given the loss of rescue in acellular implants or those containing cdc42-deficient cells, the in vivo dynamic vascular remodelling activity of the constructs appears to require functional endothelial cells. Beyond remodelling activity, a polarized endothelium prevents direct contact of materials with blood, which otherwise has shown to lead to rapid vessel occlusion $(14,40,41)$. The current proof-of-concept study used a construct containing human endothelial cells implanted in immunocompromised rodents, an approach that takes into consideration the post-surgery medications that bypass candidate patients undergo. While innate immunity is intact in these models, a fully immunocompetent host might be expected to modulate the response to the implant. Immune cells have been shown to contribute to angiogenesis, vascular remodelling, and inflammation (42), each of which could either improve or mute the therapeutic benefits of the approach. Thus, a study using autologous cells in an immunocompetent host or heterologous cells in larger immunosuppressed 
animals, is warranted to further evaluate the possible clinical outcomes of this approach. Moreover, because of the multifactorial nature of ischaemic cardiovascular diseases, the choice of animal model (small versus large animals, obesity/diabetes and aging versus no risk factors) and endpoint are considerations that could impact outcomes, and in part the lack of significant clinical successes in this arena to date speak to the uncertain nature of the extent to which preclinical models predict outcomes of revascularization therapies in humans (43). Even with these clinical development challenges met, it should be recognized that a cell-based therapy ultimately remains a resource intensive endeavour that is only considered given that acellular alternatives may not be as effective.

Although treatment of focal blockages in large arteries has largely been addressed through a combination of percutaneous interventions and surgical bypass grafts (6-14), approaches to enhance development of collateral circulation at the level of small vessels as demonstrated here could provide additional therapeutic benefit. Not only may such approaches help to address the many patients that also experience small vessel disease, but it also could be used to induce additional collaterals in at-risk patients to prophylaxis against progression of organ malperfusion. In addition, for inoperable patients (due to anatomic factors or medical comorbidities (6-10)), induction of collateral circulation could be an alternative to relieve ischaemia.

\section{Methods}

\section{Cell culture and Matrigel ${ }^{\mathrm{TM}}$ assay}

Primary human umbilical endothelial cells (HUVECs; Lonza) were cultured up to 7 passages on $0.1 \%$ (wt/vol) gelatin-coated dishes in EGM-2 (Lonza), maintained at $37^{\circ} \mathrm{C}$ in $100 \%$ humidified air containing 5\% CO2 with medium changed every other day. Certificate of analysis for source, identity and infective agents screening was provided by the vendor, and mycoplasma testing was routinely run as a lab good practice. HUVECs labeled with mCherry (generous gift from Dr. Roger Tsien) were used for ex-vivo imaging purposes.

To assess in vitro vascular network formation, HUVECs were plated onto a thin layer ( $50 \mu \mathrm{l} /$ $\mathrm{cm} 2$ ) of Matrigel $^{\mathrm{TM}}$ (Basement Membrane Matrix, BD Biosciences) at a density of 10,000 cells $/ \mathrm{cm} 2$ and cultured in EGM2 medium for 16h in presence of $10 \mu \mathrm{M}$ Y27632, $30 \mu \mathrm{M}$ ML141, $50 \mu$ M NSC23766 (Sigma).

\section{CDC42 Knock-Down}

For silencing CDC42, sixty percent confluent HUVECs were transfected for $6 \mathrm{~h}$ with $20 \mathrm{nM}$ CDC42 siRNA (Hs_CDC42_7 FlexiTube siRNA, Qiagen) or Scrambled, in a solution of Lipofectamine ${ }^{\mathrm{TM}}$ RNAiMAX (invitrogen) according to manufacturer's instructions. Expression levels of CDC42 were assessed by Real time PCR (qScript ${ }^{\mathrm{TM}}$ cDNA SuperMix, Quanta Bioscience for cDNA synthesis, and QuantiTect SYBR Green PCR Kit, Qiagen for PCR reaction) and by antibody staining (mouse anti-CDC42, Clone 44, BD Biosciences). GAPDH primers used: Fw $\left(5^{\prime}-3^{\prime}\right)$ GTCTCCTCTGACTTCAACAGCG and Rv $\left(3^{\prime}-5^{\prime}\right)$ ACCACCCTGTTGCTGTAGCCAA). Human CDC42 primers used: Fw $\left(5^{\prime}-3^{\prime}\right)$ TGACAGATTACGACCGCTGAGTT and Rv $\left(3^{\prime}-5^{\prime}\right)$ 
GGAGTCTTTGGACAGTGGTGAG). Channels within molds were seeded with transfected HUVECs and either left in culture for apoptosis and sprouting assay, or implanted in vivo in a model of hind limb ischaemia. Active caspase $3(\mathrm{Rb}$ pAb, Abcam ) staining was used to determine the percentage of apoptotic cells within channels. The growth of angiogenic sprouts from patterned vessels was tested after a four day culture in fibroblast conditioned media-EGM2 medium (1:1), followed by fixation in 4\% PFA for $30 \mathrm{~min}$ and phalloidin (Invitrogen) staining for $1 \mathrm{~h}$.

\section{D printing of carbohydrate glass patterns}

A mixture of $100 \mathrm{~g}$ isomalt, $10 \mathrm{~g}$ dextran $(86 \mathrm{kDa})$, and $60 \mathrm{ml}$ reverse osmosis water ( $\geq 18$ $\mathrm{M} \Omega$; Millipore) was warmed to $155^{\circ} \mathrm{C}$ to remove most of the water and form a liquid glass. The hot mixture was poured into a $50 \mathrm{ml}$ syringe that was maintained at $110^{\circ} \mathrm{C}$. The syringe was mounted on a ShopBot Desktop custom-modified 3D printer to use for additive layer manufacturing with an added RAMBo 1.3 (Ultimachine) circuit board. Carbohydrate-glass lattices were printed at $110^{\circ} \mathrm{C}$ under nitrogen pressure with pneumatic control through an 18-gauge (0.84 $\mathrm{mm} \mathrm{ID)} \mathrm{steel} \mathrm{nozzle,} \mathrm{vitrified} \mathrm{to} 50^{\circ} \mathrm{C}$, and then air-sprayed (Badger AirBrush Co. Model 175-7) with a $5 \mathrm{mg} \mathrm{ml}^{-1}$ solution of PDLGA (Purac) in chloroform. Glass molds were either immediately encapsulated in $\mathrm{ECM}$ or stored at $90^{\circ} \mathrm{F}$ until use to protect hygroscopic properties.

\section{Casting of vascular structures}

A fibrin gel solution $\left(10 \mathrm{mg} \mathrm{ml}^{-1}\right)$ was generated by mixing fibrinogen, thrombin $(2.5 \mathrm{U} / \mathrm{ml}$ final concentration) and phosphate-buffered saline (PBS), and then dispensed to encapsulate the printed carbohydrate glass molds. Fibrin gels were allowed to polymerize for $15 \mathrm{~min}$ at $37^{\circ} \mathrm{C}$ then placed in PBS and washed until the carbohydrate glass was completely dissolved. The encapsulated patterns were then ported at each corner using a $2 \mathrm{~mm}$ biopsy punch and the dissolved sugar solution was flushed out with PBS. A suspension of $3.5 \times 10^{6} \mathrm{cells} \mathrm{ml}^{-1}$ HUVECs in medium was injected into the ports of the hollow mold architecture and allowed to attach to channel lumens in static culture for one hour before placing on a platform rocker in a humidified $37^{\circ} \mathrm{C}$ incubator to induce gravity-driven flow overnight. In some cases, HUVECs were co-injected with $1 \mathrm{mg} / \mathrm{mL}$ type I collagen into the molds with similar bioactivity following implantation. For experiments with randomly-patterned HUVECs, cells were mixed into a bulk fibrin gel as it polymerized, and then similarly placed on a platform rocker in a humidified $37^{\circ} \mathrm{C}$ incubator overnight prior to implantation.

\section{Mouse ischemic hind limb model}

Seven to ten weeks old female nude mice (Taconic) were anesthetized with 1-3\% isoflurane at flow rate of $11 \mathrm{~min}-1$ and body temperature was maintained on a circulating heated water pad, during both the surgical procedure and Laser Doppler Imaging (LDI) monitoring. Following skin incision, the femoral neurovascular pedicle was visualized under a stereoscope and the left femoral nerve and vein were carefully separated from the femoral artery. The femoral artery abscission was executed below the deep branch by electrocauterization. In this mild moderate model of hind limb ischaemia, the perfusion of operated distal limb was assessed in the immediate post-op to be half of the contralateral. This model did not develop spontaneous limb necrosis or self-amputation for the duration of 
the study mirroring symptoms of significant ischaemia, such as ABI index and histopathological features, in patients with peripheral artery disease (PAD). Once the artery was occluded, implants were positioned with channels in parallel to the downward running femoral artery, the surgical site was sutured and inspected for infection and scarring for the duration of the study. Mice of the sham group underwent electrocauterization of femoral artery to generate ischaemia, no implant was inserted, and the site was sutured. Laser Doppler Imaging (Moor Instrument Inc.) was performed as described previously (48-50), at 16 h, day 3, day 5 after surgery. Mice were scanned at a standardized working distance of 32 $\mathrm{cm}$. Blood flow through the feet and the supine inferior limbs was documented as $2 \mathrm{D}$ colorcoded image (setting of scan modus 4-10 ms/pixel with resolution at $256 \times 256$ pixels). Results are expressed as fold increase/decrease of perfusion units (PU) with respect to the contralateral foot $(=1)$. Lamorte power calculation was adopted to estimate sample size to detect a specific effect. All data points collected were included in the analysis. Animals were randomly assigned to groups. All outcomes were assessed disregarding to group allocation. All animal procedures were performed at the Charles River campus animal Facility, Boston University, under a protocol approved by the institutional IACUC. All experiments pertaining to this investigation conformed to the "Guide for the Care and Use of Laboratory Animals," published by the US National Institutes of Health (Eighth Edition, 2011).

\section{Rat myocardium infarction model}

Athymic male RNU rats weighing 170-190g were obtained from Charles River Labs. The continuous delivery of $3 \%$ isofluorane was used to anesthetize rats. A 16-gauge angiocatheter was used for endotracheal intubation and connected to mechanical ventilation (Hallowell EMC) where 1\% isoflurane was maintained throughout the operation. A thoracotomy was performed through the left 4th intercostal space, the heart was exposed, and a 7-0 polypropylene suture was placed around the left anterior descending artery $2 \mathrm{~mm}$ below the left atrium. The suture was briefly snared to verify the size and location of myocardial ischaemia based on color change, and permanently tied down to produce a large anterolateral MI. The animals were then randomized to 3 different groups: healthy rats (no myocardial infarction, no MI), untreated operated rats injected with saline (Sham), rats implanted with patterned constructs (affixed with 7-0 polypropylene suture to the center of the infarct). The thoracotomy was closed in multiple layers and tissue adhesive (VetBond, $3 \mathrm{M})$ was applied over the incision, followed by recovery from anesthesia. Buprenorphine $(0.5 \mathrm{mg} / \mathrm{kg})$ was administered for post-operative pain control. Lamorte power calculation was adopted to estimate sample size to detect a specific effect. All data points collected were included in the analysis. Animals were randomly assigned to groups. All outcomes were assessed disregarding to group allocation. All experiments pertaining to this investigation conformed to the "Guide for the Care and Use of Laboratory Animals," published by the US National Institutes of Health (Eighth Edition, 2011). The protocol was approved by the Institutional Animal Use and Care Committee of the University of Pennsylvania.

\section{Echocardiographic and Hemodynamic Assessment}

LV geometry and function were evaluated pre-operatively and at 4 weeks in all animals. A Phillips Sonos 5500 revD ultrasound system (Philips Medical Systems, Amsterdam, ND) was used, utilizing a 12-MHz transducer at an image depth of $2 \mathrm{~cm}$. Left ventricular 
parasternal short axis 2D and M-mode images at the level of the papillary muscle were used to obtain echocardiographic data.

Four weeks after LAD ligation, all three groups of animals underwent invasive hemodynamic measurements with a pressure-volume (P-V) conductance catheter (SPR-869; Millar Instruments, Inc.). The catheter was calibrated via 5 point cuvette linear interpolation with parallel conductance subtraction by the hypertonic saline method. Rats were anesthetized as above, and the catheter was introduced into the LV utilizing a closed-chest approach via the right carotid artery. Measurements were obtained before and during inferior vena cava occlusion to produce static and dynamic P-V loops under varying load conditions. Data were recorded and analyzed with LabChart version 7 software (AD instruments). Finally, cardiac output was assessed by placing a $2.5 \mathrm{~mm}$ peri-aortic Doppler flow probe (Transonic Systems) around the ascending aorta.

\section{Visualization and functional assessment of perfusion in the implanted vasculature}

Five days following implantation, mice were anesthetized with $3 \%$ isoflurane continuously delivered and i.v. injected with $200 \mu$ of UEA-1 lectin solution (Rhodamine or FITCconjugated, Vectors Lab) or $500 \mu \mathrm{l}$ of $1 \mathrm{mg} / \mathrm{mL}$ Dextran70KDa (FITC or Texas Red conjugated, Molecular probes). Mice were kept under anesthesia (1\% isoluorane) for 15 minutes, then euthanized by cervical dislocation. Implants were harvested in $4 \%$ PFA, and either whole-mount imaged or submerged in OCT (TissueTek), frozen and stored in a $-80^{\circ} \mathrm{C}$ until imaging. Four weeks after implantation, a subset of rats was anesthetized with 3\% isoflurane continuously delivered, endotracheally intubated with a 16-gauge angio catheter connected to mechanical ventilation (Hallowell EMC). A median sternotomy was performed, exposing the heart and ascending aorta. The ascending aorta was clamped, and a 16-gauge needle was inserted into the proximal ascending aorta and connected to pressure tubing with a three way stopcock at its end. An atriotomy was made in the right atrium with scissors, and PBS was infused through the aortic needle until effluent from the right atrium was clear. Next,10cc of DiI solution (D-282, Invitrogen) was infused through the aortic needle. Hearts were explanted, submerged in OCT (TissueTek), frozen and stored at $-80^{\circ} \mathrm{C}$. Assessment for blood vessels was conducted on cryosections of 100-200 $\mu$ m thickness.

\section{Post-mortem tissue preparation, Staining and Imaging}

Animals were euthanized according to protocols approved by the institutional IACUC. Tissues and implanted constructs were dissected, OCT-embedded and snap frozen in liquid nitrogen. Histology sections (15 to $100 \mu \mathrm{m}$ thickness), whole mount preparation, and hematoxylin and eosin (H\&E) were performed by standard procedures. A solution of 5\% goat serum, $0.1 \%$ Triton X-100, in PBS $1 \mathrm{X}$ was used for the $2 \mathrm{~h}$, RT blocking prior to staining. Primary antibodies were incubated at 4 degrees $\mathrm{C}$, overnight. Rat anti-mouse Ter-119 (BD Pharmingen, 1:100), monoclonal rat anti-mouse PECAM-1 (BD Pharmingen, 1:100), sheep anti-VWF (conjugated to FITC, Abcam, 1:100), goat polyclonal anti-EphB4 (N-19, Santa Cruz, 1:100), rabbit polyclonal Ephrin B2 (Novus Biologicals, 1:100). Secondary antibodies conjugated to Alexa -488, -555, -647 from Molecular Probes were used at 1:300 dilution, 2h incubation at RT. Isolectin B4 (Vector Labs, 1:200) and Cy3 and FITC-conjugated anti-aSMA mouse mAb (Sigma Aldrich, 1:300) were used at the same 
conditions of secondary Abs. Images were acquired with an LD C-Apochromat 40×, 1.1 N.A. water-immersion objective attached to an Axiovert 200M inverted microscope (Zeiss) equipped with a CSU10 spinning disk confocal scan head (Yokogawa Electric Corp.) and an Evolve EMCCD camera (Photometrics). A DF5000B Leica fluorescent microscope was used to image $10 \mu \mathrm{m}$ thick heart sections. Image processing and analysis was performed with by Fiji/ImageJ software.

\section{Statistical analysis}

Normality of data distribution was evaluated by Shapiro-Wilk test. Mann-Whitney U test was used accordingly to determine statistical significant differences between groups. Statistical significance threshold for this test was set at $\mathrm{p} \leq 0.05$. For data assumed to be normally distributed, either a two sided T-test or a one-way ANOVA with Bonferroni posthoc correction were used, with statistical significance threshold set at $p \leq 0.01$.

\section{Supplementary Material}

Refer to Web version on PubMed Central for supplementary material.

\section{Acknowledgments}

We thank J. Eyckmans, R. Chaturvedi, and M. Shockley for helpful discussions. This work was supported in part by grants from the National Institutes of Health (EB00262, EB08396, HL118851), the Biological Design Center of Boston University, and the BU-Coulter Foundation Translational Partnership Program. D.C. is supported by the National Science Foundation, and K.O. by the American Heart Association Grant-in-Aid (16GRNT27090006). YJW is supported by NIH grant 1R01 (HL089315-01). JWM is supported by AHA (12POST11620024).

\section{References}

1. CDC, NCHS. Underlying Cause of Death 1999-2013 on CDC WONDER Online Database, released 2015. Data are from the Multiple Cause of Death Files, 1999-2013, as compiled from data provided by the 57 vital statistics jurisdictions through the Vital Statistics Cooperative Program. [Accessed Feb. 3, 2015]

2. CDC. Deaths, Percent of Total Deaths, and Death Rates for the 15 Leading Causes of Death: United States and Each State, 1999-2014.

3. Mozaffarian D, et al. American Heart Association Statistics Committee and Stroke Statistics Subcommittee Heart disease and stroke statistics-2015 update: a report from the American Heart Association. Circulation. 2015; 131:29-322.

4. Fryar CD, Chen TC, Li X. Prevalence of uncontrolled risk factors for cardiovascular disease: United States, 1999-2010. NCHS Data Brief. 2012; 103:1-8.

5. Fowkes FG, et al. Comparison of global estimates of prevalence and risk factors for peripheral artery disease in 2000 and 2010: a systematic review and analysis. Lancet. 2013; 382:1329-1340. [PubMed: 23915883]

6. Hall MJ, DeFrances CJ, Williams SN, Golosinskiy A, Schwartzman A. National Hospital Discharge Survey: 2007 summary. Natl Health Stat Report. 2010; 29:1-20.

7. Conte MS. Bypass versus Angioplasty in Severe Ischaemia of the Leg (BASIL) and the (hoped for) dawn of evidence-based treatment for advanced limb ischaemia. J Vasc Surg. 2010; 51:69S-75S. [PubMed: 20435263]

8. Salacinski HJ, et al. The mechanical behavior of vascular grafts: a review. J Biomater Appl. 2001; 15:241-278. [PubMed: 11261602]

9. Slovut DP, Lipsitz EC. Surgical technique and peripheral artery disease. Circulation. 2012; 126:1127-1138. [PubMed: 22927475] 
10. Kakkar AM, Abbott JD. Percutaneous versus surgical management of lower extremity peripheral artery disease. Curr Atheroscler Rep. 2015; 17:479. [PubMed: 25612856]

11. Kappetein AP, Van Mieghem NM, Head SJ. Revascularization options: coronary artery bypass surgery and percutaneous coronary intervention. Cardiol Clin. 2014; 32:457-461. [PubMed: 25091970]

12. L'Heureux N, et al. Human tissue-engineered blood vessels for adult arterial revascularization. Nat Med. 2006; 12:361-365. [PubMed: 16491087]

13. Dahl SL, et al. Readily available tissue-engineered vascular grafts. Sci Transl Med. 2011; 3:68ra9.

14. Quint C, et al. Decellularized tissue-engineered blood vessel as an arterial conduit. Proc Natl Acad Sci U S A. 2011; 108:9214-9219. [PubMed: 21571635]

15. Henry TD, et al. The VIVA trial: Vascular endothelial growth factor in Ischaemia for Vascular Angiogenesis. Circulation. 2003; 107:1359-1365. [PubMed: 12642354]

16. Simons M, et al. Pharmacological treatment of coronary artery disease with recombinant fibroblast growth factor-2: double-blind, randomized, controlled clinical trial. Circulation. 2002; 105:788793. [PubMed: 11854116]

17. Giacca M, Zacchigna S. VEGF gene therapy: therapeutic angiogenesis in the clinic and beyond. Gene Ther. 2012; 19:622-629. [PubMed: 22378343]

18. Ferrara N. The role of vascular endothelial growth factor in pathological angiogenesis. Breast Cancer Res Treat. 1995; 36:127-137. [PubMed: 8534862]

19. Peters MC, Polverini PJ, Mooney DJ. Engineering vascular networks in porous polymer matrices. J Biomed Mater Res. 2002; 60:668-678. [PubMed: 11948526]

20. Ehrbar M, et al. Cell-demanded liberation of VEGF121 from fibrin implants induces local and controlled blood vessel growth. Circ Res. 2004; 94:1124-1132. [PubMed: 15044320]

21. Phelps EA, Landázuri N, Thulé PM, Taylor WR, García AJ. Bioartificial matrices for therapeutic vascularization. Proc Natl Acad Sci U S A. 2010; 107:3323-3328. [PubMed: 20080569]

22. Sadr N, et al. SAM-based cell transfer to photopatterned hydrogels for microengineering vascularlike structures. Biomaterials. 2011; 32:7479-7490. [PubMed: 21802723]

23. Gupta R, Tongers J, Losordo DW. Human studies of angiogenic gene therapy. Circ Res. 2009; 105:724-736. [PubMed: 19815827]

24. Koike N, et al. Tissue engineering: creation of long-lasting blood vessels. Nature. 2004; 428:138139. [PubMed: 15014486]

25. Au P, Tam J, Fukumura D, Jain RK. Bone marrow-derived mesenchymal stem cells facilitate engineering of long-lasting functional vasculature. Blood. 2008; 111:4551-4558. [PubMed: 18256324]

26. Kusuma S, et al. Self-organized vascular networks from human pluripotent stem cells in a synthetic matrix. Proc Natl Acad Sci U S A. 2013; 110:12601-12606. [PubMed: 23858432]

27. Levenberg S, et al. Engineering vascularized skeletal muscle tissue. Nat Biotechnol. 2005; 23:879_ 884. [PubMed: 15965465]

28. Baranski JD, et al. Geometric control of vascular networks to enhance engineered tissue integration and function. Proc Natl Acad Sci U S A. 2013; 110:7586-7591. [PubMed: 23610423]

29. Miller JS, et al. Rapid casting of patterned vascular networks for perfusable engineered threedimensional tissues. Nat Mater. 2012; 11:768-774. [PubMed: 22751181]

30. Hiesinger W, et al. Computational protein design to reengineer stromal cell-derived factor-1a generates an effective and translatable angiogenic polypeptide analog. Circulation. 2011; 124:S1826. [PubMed: 21911811]

31. MacArthur JW, et al. Mathematically engineered stromal cell-derived factor-1a stem cell cytokine analog enhances mechanical properties of infarcted myocardium. J Thorac Cardiovasc Surg. 2013; 145:278-284. [PubMed: 23244259]

32. MacArthur JW, et al. Sustained release of engineered stromal cell-derived factor 1-a from injectable hydrogels effectively recruits endothelial progenitor cells and preserves ventricular function after myocardial infarction. Circulation. 2013; 128:S79-86. [PubMed: 24030424] 
33. Shudo Y, et al. A Tissue-Engineered Chondrocyte Cell Sheet Induces Extracellular Matrix Modification to Enhance Ventricular Biomechanics and Attenuate Myocardial Stiffness in Ischaemic Cardiomyopathy. Tissue Eng Part A. 2015; 19-20:2515-2525.

34. Rodell CB, et al. Shear-Thinning Supramolecular Hydrogels with Secondary Autonomous Covalent Crosslinking to Modulate Viscoelastic Properties In Vivo. Adv Funct Mater. 2015; 25:636-644. [PubMed: 26526097]

35. Murray CD. The Physiological Principle of Minimum Work: I. The Vascular System and the Cost of Blood Volume. Proc Natl Acad Sci U S A. 1926; 12:207-214. [PubMed: 16576980]

36. Kassab GS. Scaling laws of vascular trees: of form and function. Am J Physiol Heart Circ Physiol. 2006; 290:H894-903. [PubMed: 16143652]

37. Atala, A., Yoo, JJ., editors. Essentials of 3D Biofabrication and Translation. London (UK): Elsevier/Academic Press; 2015. p. 179-186.

38. Visconti RP, et al. Towards organ printing: engineering an intra-organ branched vascular tree. Expert Opin Biol Ther. 2010; 10:409-420. [PubMed: 20132061]

39. Sydney Gladman A, Matsumoto EA, Nuzzo RG, Mahadevan L, Lewis JA. Biomimetic 4D printing. Nat Mater. 2016; 15:413-418. [PubMed: 26808461]

40. Moroni F, Mirabella T. Decellularized matrices for cardiovascular tissue engineering. Am J Stem Cells. 2014; 13:1-20.

41. Sooppan R, et al. In Vivo Anastomosis and Perfusion of a Three-Dimensionally-Printed Construct Containing Microchannel Networks. Tissue Eng Part C Methods. 2016; 22:1-7. [PubMed: 26414863]

42. Jaipersad AS, Lip GY, Silverman S, Shantsila E. The role of monocytes in angiogenesis and atherosclerosis. J Am Coll Cardiol. 2014; 63:1-11. [PubMed: 24140662]

43. Waters RE, Terjung RL, Peters KG, Annex BH. Preclinical models of human peripheral arterial occlusive disease: implications for investigation of therapeutic agents. J Appl Physiol (1985). 2004; 97:773-80. [PubMed: 15107408]

44. Hall MJ, et al. National Hospital Discharge Survey: 2007 summary. Natl Health Stat Report. 2010; 24:1-20.

45. Norgren L, et al. Inter-Society Consensus for the Management of Peripheral Arterial Disease (TASC II). J Vasc Surg. 2007; 45:S5-67. [PubMed: 17223489]

46. Belch JJ, et al. Critical issues in peripheral arterial disease detection and management: a call to action. Arch Intern Med. 2003; 163:884-892. [PubMed: 12719196]

47. Al Amjad, M. Peripheral artery disease. Cleveland Clinic Center for Continuing Education; 2009.

48. Hoefer IE, et al. Arteriogenesis proceeds via ICAM-1/Mac-1- mediated mechanisms. Circ Res. 2004; 94:1179-1185. [PubMed: 15059933]

49. Mirabella T, Cilli M, Carlone S, Cancedda R, Gentili C. Amniotic liquid derived stem cells as reservoir of secreted angiogenic factors capable of stimulating neo-arteriogenesis in an ischaemic model. Biomaterials. 2011; 32:3689-3699. [PubMed: 21371750]

50. Mirabella T, et al. Proangiogenic soluble factors from amniotic fluid stem cells mediate the recruitment of endothelial progenitors in a model of ischaemic fasciocutaneous flap. Stem Cells Dev. 2012; 21:2179-2188. [PubMed: 22225409] 
a

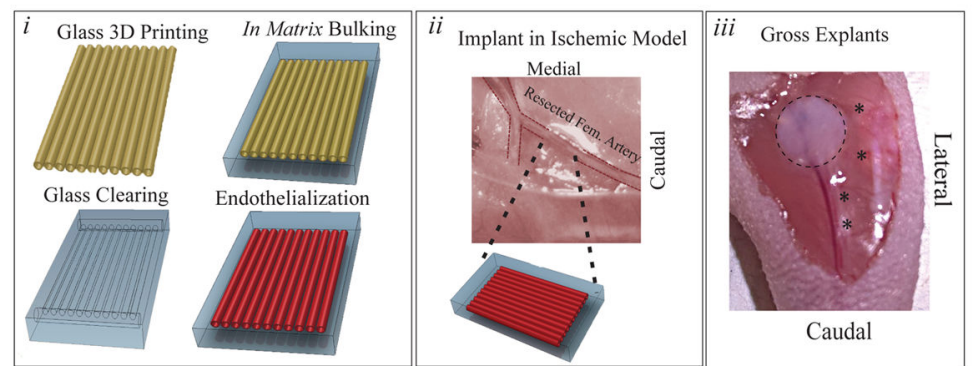

b
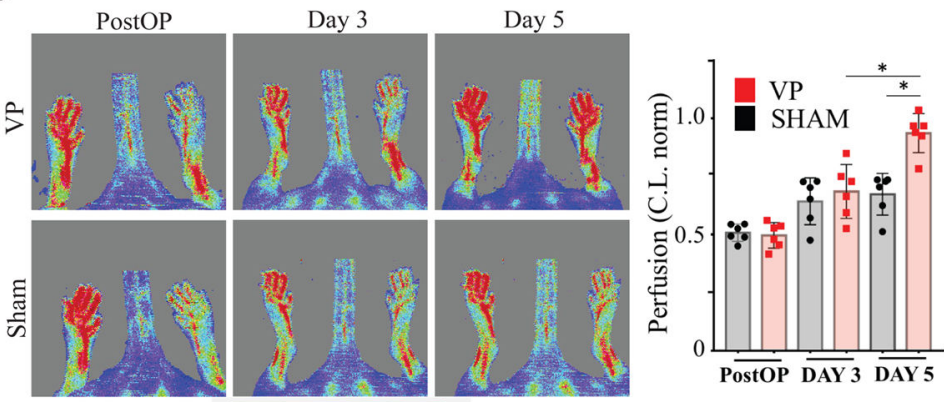

c

d
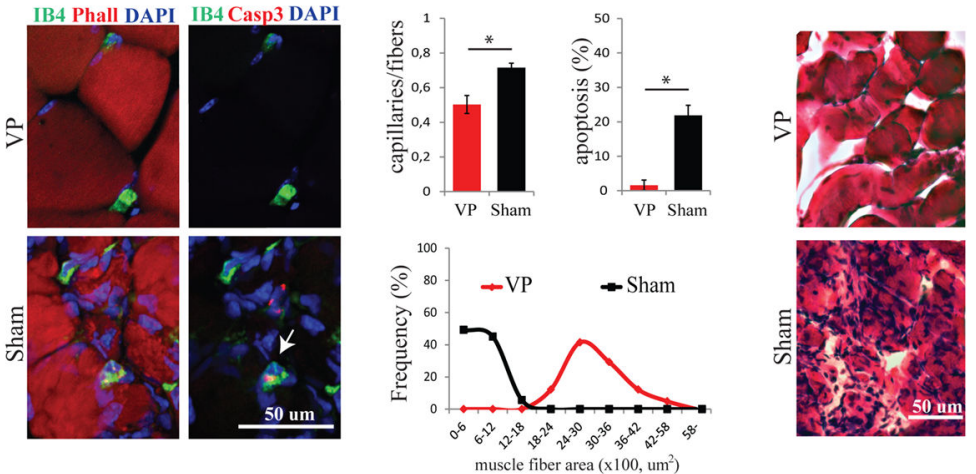

Fig. 1. Fabricated vascular patches rescue perfusion in hind limb ischaemia

(a) Schematic of VP (vascular patches) fabrication (i). Carbohydrate glasses are 3D printed as parallel filament. After PDGLA-coating and fibrin $(10 \mathrm{mg} / \mathrm{ml})$ bulking, the sacrificial sugar are removed by overnight washings in PBS. The remaining channels are endothelialized by flowing HUVECs and shear-conditioning the confluent monolayer for an overnight, before implantation in ischaemic hind limb mice. In ii), representation of the patches implantation site within the cauterized femoral artery space. In iii, representation of patches retrieved after 5 days from implantation (stars mark collateral arteries and veins). (b) Laser Doppler Imaging (Moor Instrument ${ }^{\mathrm{TM}}$ ) of distal limbs over time (post-surgery, day 3 and day5) in the two groups (VP vs Sham). Histograms for quantification of perfusion levels of the ischaemic limb. Perfusion units (P.U.) of ischaemic limb were normalized to P.U. of contralateral not ischaemic limb (C.L. norm, considered=1) and expressed as fold change. Data expressed as mean \pm S.D. $\mathrm{N}=6$ animals per group. T-test for comparison between two groups. * p-value $<0.01$. (c) Staining of Gastrocnemius muscle (GC) sections (25 um thick) with phalloidin (cytoarchiteture), IB4 (capillaries) and activated caspase 3 (apoptosis). 
Representative images (Spin Disk Confocal, 20um stack) for the two groups (VP vs Sham). Arrow points at apoptotic EC. (d) Quantifications of capillary density (\#vessels / \#muscle fibers), capillary degeneration (\% apoptotic ECs in capillaries) and muscle atrophy (distribution of muscle fiber area expressed as percentage of frequency for each area subgroup, with range 0-60 equal to 0-6000 $\mathrm{um}^{2}$ ) in the GC of the two groups (VP vs Sham). Total number of capillaries analyzed is 55 and 211 respectively for VP and Sham group. Data expressed as mean \pm S.E.M. Three animals per group, three GC sections per mouse. Mann-Whitney $U$ test for comparison between two groups. * p-value $<0.02$. (e) Hematoxilyn\&Eosin (H\&E) staining of 15 um sections of GC muscles in the two groups (VP vs Sham) revealing hypercellularization and fibrosis initiation in the not implanted mice. 
$\mathbf{a}$
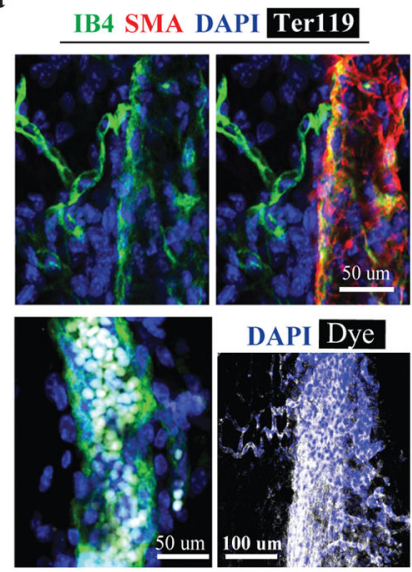

c
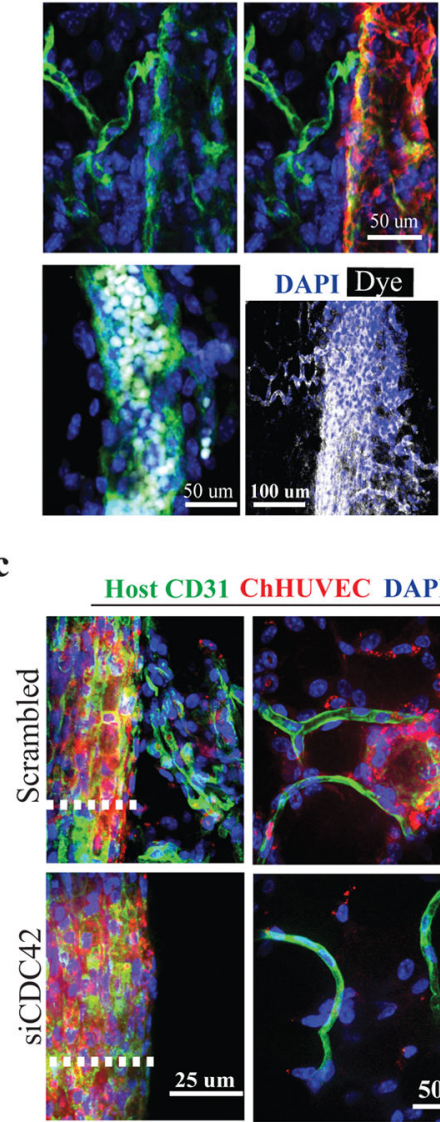

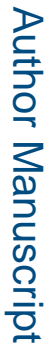

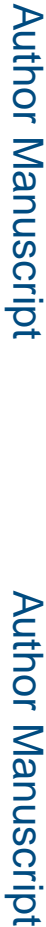

$\mathbf{e}$ b

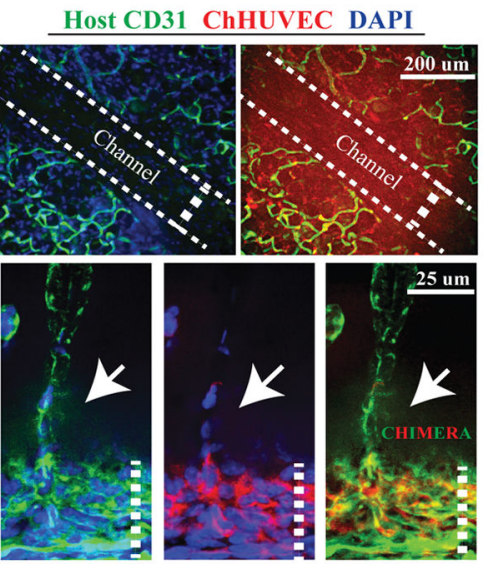

d
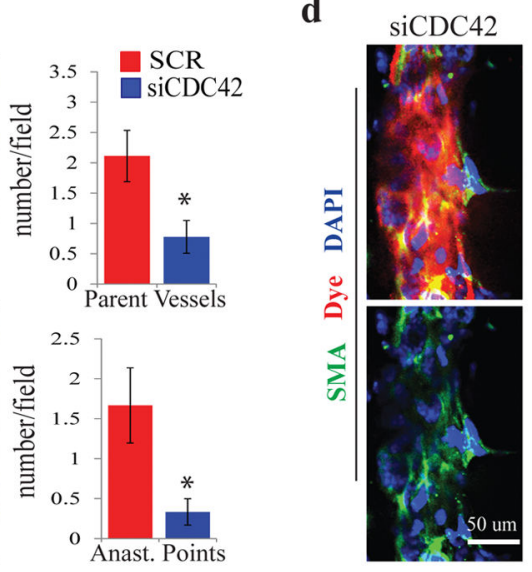

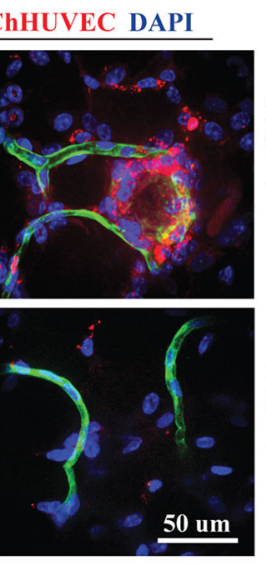

Day 5
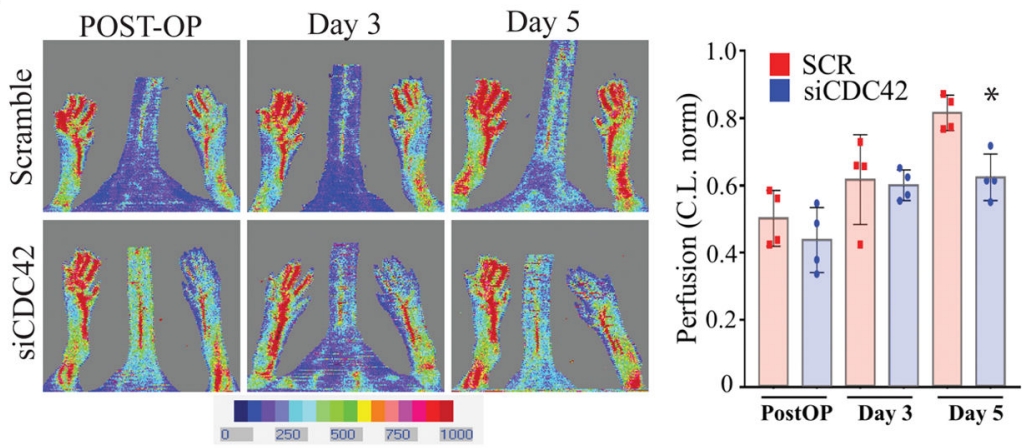

Fig. 2. Host-driven biointegration of grafts drives perfusion of ischaemic limb

(a) VP explanted at day 5 are stained for Isolectin B4 (IB4) labeling both host capillary and VP endothelium), mouse specific smooth muscle actin-alpha (SMA) labeling host-derived smooth muscle cells, and Ter119 labeling red blood cells. The lower right panel shows a channel perfused by host vasculature as demonstrated by dye localization immediately following systemic perfusion of dye-labeled dextran (b-c) Explanted VP at day 5 are stained for host vessels (mouse CD31). Endogenous red fluorescence of implanted mCherry HUVECs is also shown. In (b), upper panels show a low magnification of a channel (dashed lines) within the VP implant. Lower panel shows the high magnification of the channel, 
which is chimeric (wall covered by both Host CD31 and implanted mCherry HUVECs) and anastomosed to a host capillary (arrow). In (c), 5 days ex vivo VP (scrambled and silenced for CDC42), with left panels showing the patterned channels (dashed lines) as mosaic multicellular composites (endogenous fluorescence for implanted mCherry-HUVECs and mouse CD31-stained host ECs); and panels on the right showing anastomosis between host capillaries and parental VP-derived vessels (ChHUVECs). In histograms, the number of VPderived vessels (Parent vessels), and the number of anastomotic points between parent and host vessels was quantified in 9 fields from VP explanted from 3 mice per group (SCR vs siCDC42). Data expressed as mean \pm S.E.M. Mann-Whitney U test for comparison between two groups. * p-value < 0.04. In (d), CDC42-silenced VP explanted at day 5 were stained with mouse specific smooth muscle actin-alpha (SMA) labeling host-derived smooth muscle cells, and with UAE-1 lectin, as a systemically injected dye labeling human endothelial cells of the implanted channels to demonstrate vascular integrity and function. Panels a, b, c, d are representative $100 \mu \mathrm{m}$ stack images. In (e), Laser Doppler Imaging (Moor Instrument ${ }^{\mathrm{TM}}$ ) of distal limbs over time (post-surgery, day 3 and day5) in the two groups: control VP (scrambled) versus VP where CDC42 has been knocked down (siCDC42). Histograms for quantification of perfusion levels of the ischaemic limb. Perfusion units (P.U.) of ischaemic limb were normalized to P.U. of contralateral non ischaemic limb (C.L. norm, considered=1) and expressed as fold change. Data expressed as mean \pm S.D. $N=4$ animals per group. T-test for comparison between two groups. ${ }^{*}$ p-value $<0.05$. 

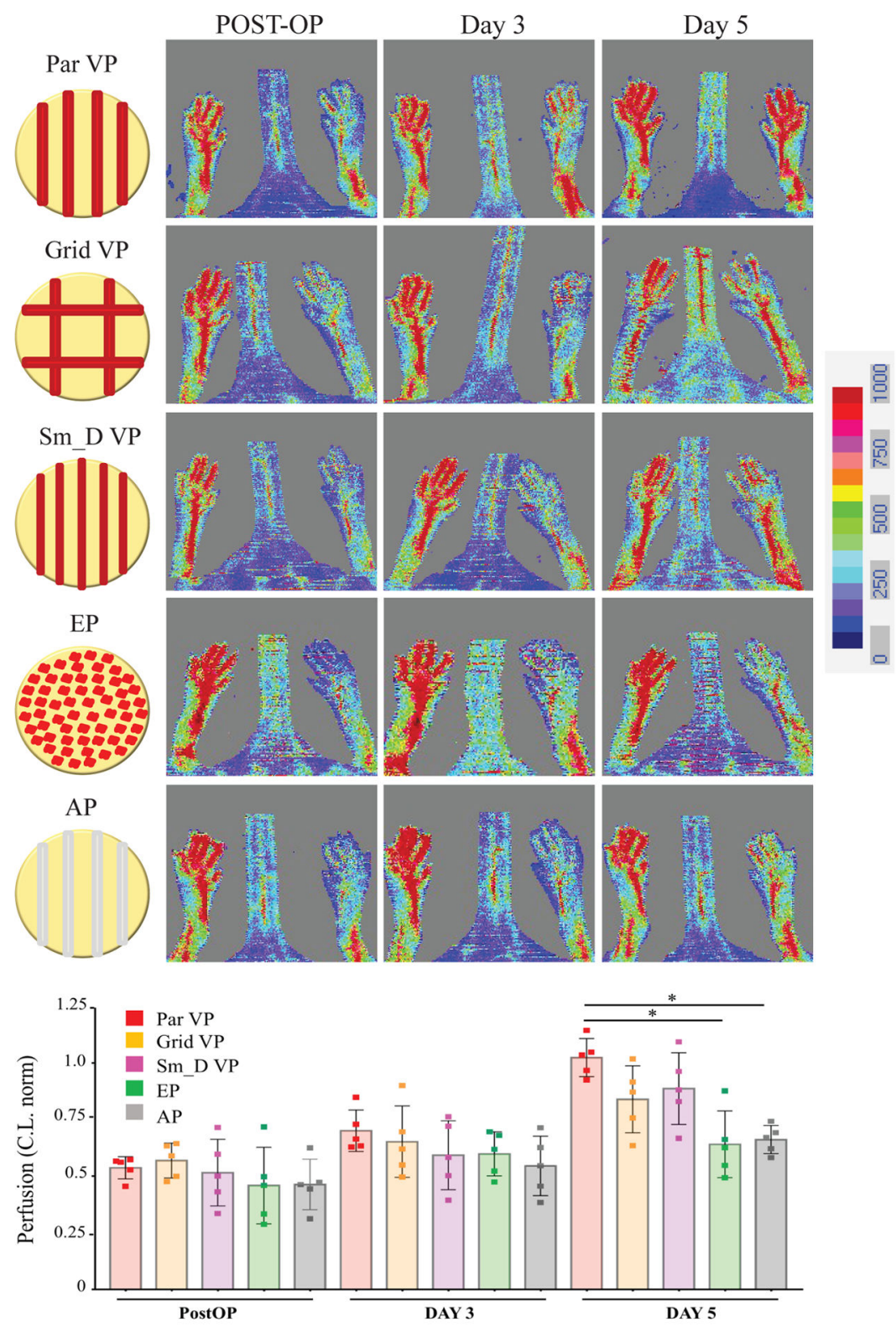

Fig. 3. Geometric patterning within vascular patches impacts perfusion performance Laser Doppler Imaging of distal limbs over time (post-surgery, day 3 and day 5) in the five groups: vascular patches where channels were oriented parallel to each other (Par VP), or orthogonally to each other to form a grid (Grid VP), vascular patches with parallel channels of smaller diameter (Sm-D VP), endothelial patches where HUVECs were randomly embedded into fibrin matrix (EP), and acellular patches (AP) containing patterned not endothelialized channels. Histograms for quantification of perfusion levels of the ischaemic limb. Perfusion units (P.U.) of ischaemic limb were normalized to P.U. of contralateral not ischaemic limb (C.L. norm, considered=1) and expressed as fold change. Data expressed as mean \pm S.D. $\mathrm{N}=5$ animals per group. Anova test with Bonferroni correction for comparison between groups. $* *$ p-value $<0.01$. 
a

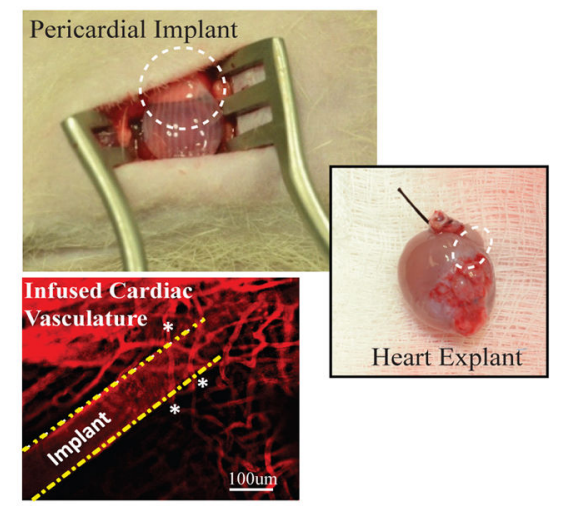

c
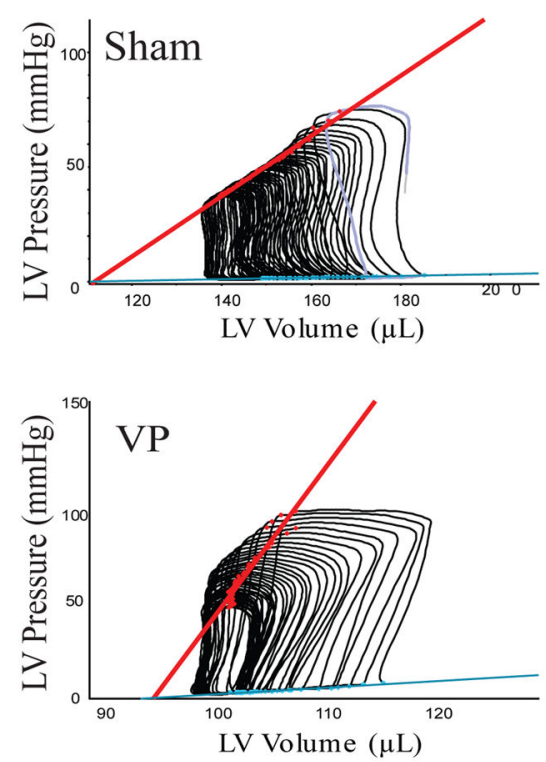

b
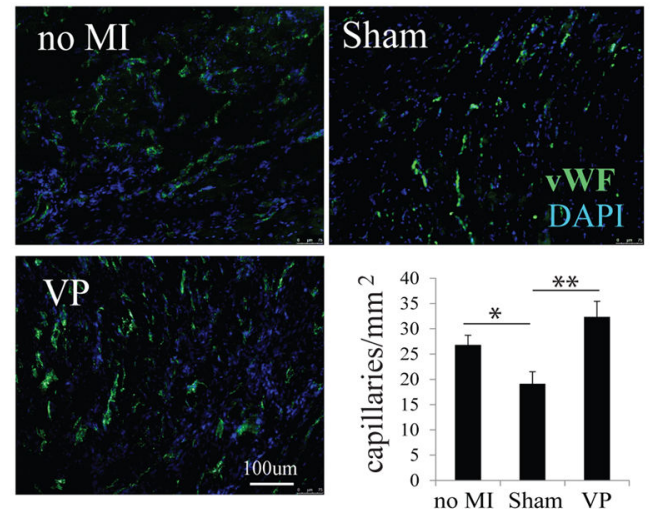

d

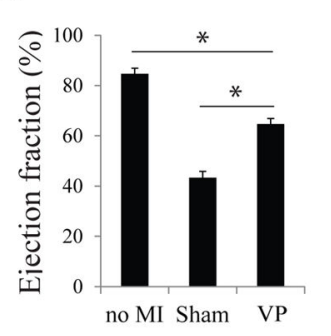

e

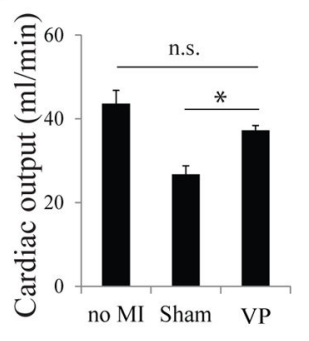

f

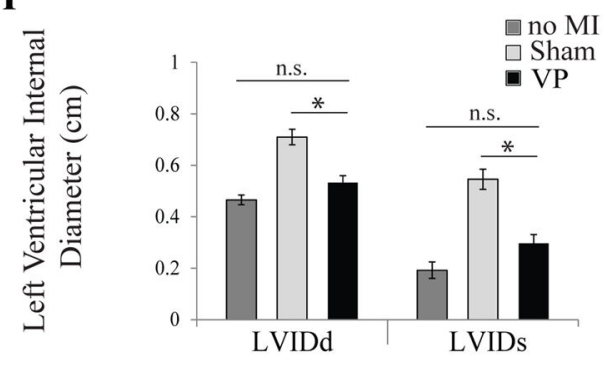

Fig. 4. Fabricated vascular patches rescue cardiac function after myocardial infarction in rats (a) Angled view of a rat undergoing implantation of vascular patches (VP, dashed circle), placed on the left ventricle of the infarcted heart. An explanted heart shows the vascularized region where the implant was placed 4 weeks prior (Inset, dashed circle). The DiI infused vasculature within the explanted patch is showed in red, with channel borders marked with dashed lines and point of connections between the channel and capillaries marked by stars. (b) Immunofluorescence images of explanted heart sections (15um thick) stained for vWF (green). The relative histogram shows vascular density for the indicated groups (no MI, sham, and VP groups) quantified as number of vWF-positive capillaries per $\mathrm{mm}^{2}$ of cardiac tissue. Heart capillaries were quantified in 3 fields per heart, from 8 explanted hearts in Sham group, and 10 explanted hearts in noMI and VP groups. $\mathrm{N}=8-10$. Data expressed as mean \pm S.E.M. Mann-Whitney $U$ test for comparison between two groups. * p-value $<0.03$, $* *$ p-value $<0.01$. (c) Representative pressure-volume loop for infarcted hearts that received either no treatment or VP. (d) Ejection fraction of infarcted hearts in the no MI, sham and VP groups. (e) Cardiac output of infarcted hearts in the no MI, sham and VP groups. (f) Left 
ventricular internal dimension for hearts in diastole (LVIDd) and systole (LVIDs) in the no MI, sham and VP groups. In D, E, F, data are expressed as mean \pm S.E.M. One way ANOVA followed by Bonferroni correction was performed to seek significance between groups, with noMI group containing 11 rats, and Sham and VP groups containing 13 rats. $\mathrm{N}=11-13$. * indicates p-value $<0.01$, while "n.s." refers to no significant difference between groups. 